\title{
Impact of a hollow density profile on turbulent particle fluxes: Gyrokinetic and fluid simulations
}

\author{
D. Tegnered, ${ }^{\text {a) }}$ M. Oberparleiter, P. Strand, and H. Nordman \\ Department of Earth and Space Sciences, Chalmers University of Technology, SE-412 96 Göteborg, Sweden
}

(Received 4 March 2017; accepted 9 June 2017; published online 29 June 2017)

\begin{abstract}
Hollow density profiles may occur in connection with pellet fuelling and $\mathrm{L}$ to $\mathrm{H}$ transitions. A positive density gradient could potentially stabilize the turbulence or change the relation between convective and diffusive fluxes, thereby reducing the turbulent transport of particles towards the center, making the pellet fuelling scheme inefficient. In the present work, the particle transport driven by Ion Temperature Gradient/Trapped Electron (ITG/TE) mode turbulence in hollow density profiles is studied by fluid as well as gyrokinetic simulations. The fluid model used, an extended version of the Weiland transport model, Extended Drift Wave Model (EDWM), incorporates an arbitrary number of ion species in a multi-fluid description and an extended wavelength spectrum. The fluid model, which is fast and hence suitable for use in predictive simulations, is compared to gyrokinetic simulations using the code GENE. Typical tokamak parameters are used based on the Cyclone Base Case. Parameter scans in key plasma parameters like plasma $\beta$, $R / L_{T}$, and magnetic shear are investigated. In addition, the effects of a fast species are studied and global ITG simulations in a simplified physics description are performed in order to investigate nonlocal effects. It is found that $\beta$ in particular, has a stabilizing effect in the negative $R / L_{n}$ region. Both nonlinear GENE and EDWM simulations show a decrease in inward flux for negative $R / L_{n}$ and a change in the direction from inward to outward for positive $R / L_{n}$. Moreover, the addition of fast particles was shown to decrease the inward main ion particle flux in the positive gradient region further. This might have serious consequences for pellet fuelling of high $\beta$ plasmas. Additionally, the heat flux in global ITG turbulence simulations indicates that nonlocal effects can play a different role from usual in connection with pellet fuelling. Published by AIP Publishing.

[http://dx.doi.org/10.1063/1.4990078]
\end{abstract}

\section{INTRODUCTION}

The characteristics of particle transport in regions of hollow density profiles are important issues for fusion plasmas. For example, reactor grade plasmas will likely be fuelled by pellet injection that will transiently perturb the density and temperature profiles, making the density profile flat or hollow. A similar effect on the density profile may occur in connection with the L-mode to H-mode transition. Hence, it is important to study the microstability and transport properties in regions with a hollow density profile. In a microstability study of pellet fuelled discharges in $\mathrm{MAST}^{1}$ using the local gyrokinetic code GS2 (Ref. 2) in the linear mode, a stabilization of all modes was found in the negative $R / L_{n}$ region as a result of the reduction in $\eta_{i}$ and $\eta_{e}$, as well as a high sensitivity to the rapid change in plasma $\beta$ and collisionality that was caused by the pellet ablation. In Ref. 3, a stabilizing effect was also found for pellet fuelled discharges at JET. The linear growth rates as well as the nonlinear heat fluxes were shown to be reduced in the positive gradient region. The quasilinear gyrokinetic code QuaLiKiz ${ }^{4}$ was used in Ref. 5 to study the microturbulence during the $\mathrm{L}$ to $\mathrm{H}$ transition; it was shown that the TE mode was stabilized and that the particle flux was highly sensitive to the sign of $R / L_{n}$ as well as the collisionality and $R / L_{T}$. In predictive modelling

\footnotetext{
a)tegnered@chalmers.se
}

of pellet fuelled L-mode discharges using the Weiland model, it was found that an anomalous pinch was necessary in order to reproduce the experimental results. ${ }^{6}$

In the present study, the effects of a positive density gradient $\left(R / L_{n}=R / L_{n_{i}}=R / L_{n_{e}}<0\right.$ where $\left.R / L_{n}=-R \nabla n / n\right)$ on the Ion Temperature Gradient/Trapped Electron (ITG/ TE) stability and particle transport for general tokamak parameters are investigated. The gyrokinetic code $\mathrm{GENE}^{7}$ is used in local and global modes to study the transport due to ITG/TE mode $\mathrm{e}^{8-14}$ turbulence. Parameter scans in plasma $\beta$, $R / L_{T}, \hat{s}, T_{i} / T_{e}$, and collisionality around those of the Cyclone Base Case (CBC) ${ }^{15}$ are used. The CBC parameters are shown in Table I. The linear and nonlinear GENE results are compared to the results from a fluid model, an extended version of the Weiland ${ }^{16}$ transport model, the Extended Drift Wave Model (EDWM). ${ }^{17}$ Fast ions have been shown to stabilize the ITG mode. ${ }^{18}$ In the present paper, the effect of fast particles in the positive gradient region is studied by adding a high temperature kinetic species with a Maxwellian background distribution. Since $R / L_{n}$ can vary over short distances during pellet fuelling, the role of nonlocal effects is also investigated.

The outline of this paper is as follows. In Sec. II, the simulation setups are described, followed by a linear stability analysis in Sec. III where the growth rates and real frequencies are compared in scans over $R / L_{n}$. This is followed by nonlinear analysis in Sec. IV, where the particle fluxes are 
TABLE I. Typical CBC parameters.

\begin{tabular}{lc}
\hline \hline Parameter & Value \\
\hline$q_{0}$ & 1.4 \\
$\hat{s}$ & 0.8 \\
$B_{0}$ & $3.1 \mathrm{~T}$ \\
$r / R$ & 0.18 \\
$R$ & $1.65 \mathrm{~m}$ \\
$T_{e}=T_{i}$ & $2.85 \mathrm{keV}$ \\
$n_{e}=n_{i}$ & $3.5 \times 10^{19} \mathrm{~m}^{-3}$ \\
$R / L_{T}=R / L_{T_{i}}=R / L_{T_{e}}$ & 6.96 \\
\hline
\end{tabular}

investigated. In Sec. V, the effects of adding fast particles are explored, and in Sec. VI, global simulations are used to investigate the role of nonlocal effects. Finally, in Sec. VII, some concluding remarks are given.

\section{GENE AND EDWM SIMULATION SETUP}

GENE solves the nonlinear gyrokinetic Vlasov equations together with Maxwell's equations in order to find the distribution functions of the species, $f\left(\mathbf{R}, v_{\|}, \mu, t\right)$, the electrostatic potential, $\phi(\mathbf{x}, t)$, and the parallel components of the magnetic vector potential and magnetic field, $A_{\|}(\mathbf{x}, t)$ and $B_{\|}(\mathbf{x}, t)$. It is a Eulerian $\delta f$-type code where the coordinate system is aligned to the background magnetic field with $x$ being the radial coordinate, $y$ being the binormal coordinate, and $z$ being the parallel coordinate. Both linear and nonlinear simulations are performed with kinetic ions and electrons. In the collisional cases, the collisions are modelled using a linearised Landau-Boltzmann collision operator. ${ }^{19}$ The pressure gradient, as used in the calculation of the curvature and $\nabla B$ drift, is set to be consistent with the density and temperature gradients and the plasma $\beta$. In this work, a circular geometry model neglecting the Shafranov shift is used.

EDWM is an extended version of the Weiland ${ }^{16,20}$ model which incorporates an arbitrary number of ion species in a multi-fluid description and an extended wavelength spectrum. Here, the wavelength is fixed at $k_{y} \rho_{s}=0.2$ for the comparison to nonlinear GENE since it is the wavelength corresponding to the largest fluxes. For the linear comparisons, the wavelength is fixed at $k_{y} \rho_{s}=0.3$ since it is the wavelength corresponding to the largest ITG growth rates and $\rho_{s}$ is the sound Larmor radius defined as $\rho_{s}=c_{s} /$ $\left(e B / m_{s} c\right)$. In the fluid model, the perturbations in density, parallel velocity, and temperature for each species are described by the continuity, parallel momentum, and energy equations, including Finite Larmor Radius (FLR) effects for the main ions. The densities of the ions, trapped, and free electrons are coupled through the quasineutrality condition. The free electrons are assumed to be Boltzmann distributed in the electrostatic case. A weakly ballooning eigenfunction is assumed whereby the parallel wavenumber $k_{\|}$, the perpendicular wavenumber $k_{\perp}$ and the magnetic drift $\omega_{D j}$ are replaced by averages over the weakly ballooning eigenfunction. The particle and heat fluxes are then calculated assuming linear relations between the field quantities combined with a modified mixing length estimate. ${ }^{16}$

\section{LINEAR RESULTS}

The computational parameters used in the linear GENE simulations are a resolution of $48 \times 12$ in the parallel and normal direction with 32 grid points in the parallel velocity direction and 24 magnetic moments. An initial value solver is used. Two main cases are simulated linearly: the mixed case with $R / L_{T_{e}}=R / L_{T_{i}}=6.96$, and a case where the TE mode is primarily unstable with $R / L_{T_{e}}=6.96, R / L_{T_{i}}=0.0$.

In Fig. 1, the linear growth rates, real frequencies, and normalized particle fluxes $\Gamma / \delta n^{2}$ are shown for the mixed case as a function of the normalized density gradient scale length $R /$ $L_{n}$ and the normalized poloidal wave number $k_{y} \rho_{s}$. In the mixed case, the ITG mode dominates roughly in the area $k_{y} \rho_{s}<0.5$ and $R / L_{n}<4$ and the area $k_{y} \rho_{s}>0.5$ and $R / L_{n}>6$ as indicated by the positive values of the real frequencies in Fig. 1(b). Here, a positive $\omega_{r}$ means that the mode drifts in the ion diamagnetic direction. The TE mode is dominant elsewhere, as indicated by the negative real frequency. In Fig. 1(c), the direction of the particle flux is indicated at different wave numbers and density gradients in the mixed case. Positive values correspond to an outward particle flux while negative correspond to an inward one. The gradient of zero particle flux, representing the peaking factor, is indicated. It increases with the wave number from $P F \approx 3$ at $k_{y} \rho_{s}=0.2$ to 6 at $k_{y} \rho_{s}=1.2$.

In Fig. 2, the eigenvalue spectra for the TE case are examined. Here, the TE mode dominates everywhere and is



(a)



(b)

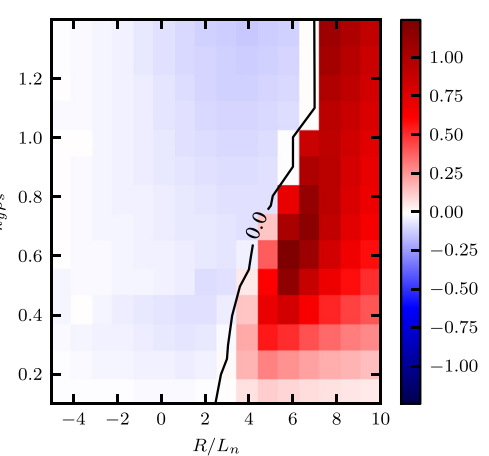

(c)

FIG. 1. GENE eigenvalues as a function of $k_{y} \rho_{s}$ and $R / L_{n_{e}}=R / L_{n_{i}}$ for the mixed case where $R / L_{T_{e}}=R / L_{T_{i}}=6.96$. (a) Linear growth rate, $\gamma\left[c_{s} / R\right]$. (b) Linear real frequency, $\omega_{r}\left[c_{s} / R\right]$. (c) Normalized flux, $\Gamma / \delta n^{2}$. 


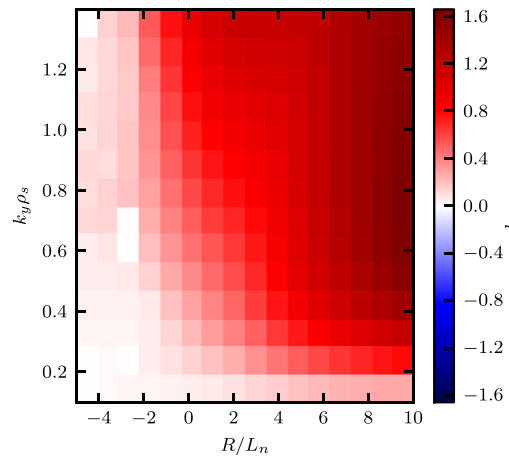

(a)

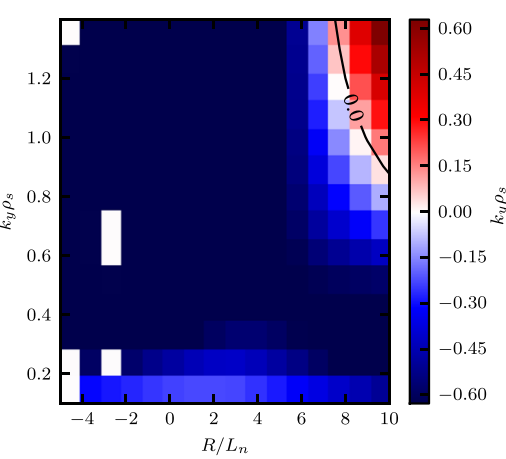

(b)

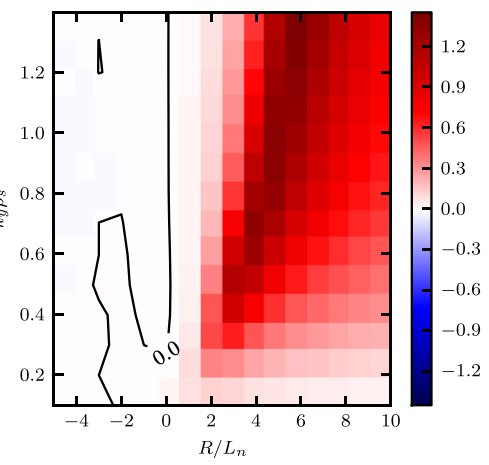

(c)

FIG. 2. GENE eigenvalues as a function of $k_{y} \rho_{s}$ and $R / L_{n_{e}}=R / L_{n_{i}}$ for the TE case where $R / L_{T_{e}}=6.96, R / L_{T_{i}}=0.0$. (a) Linear growth rate, $\gamma\left[c_{s} / R\right]$. (b) Linear real frequency, $\omega_{r}\left[c_{s} / R\right]$. (c) Normalized flux, $\Gamma / \delta n^{2}$.

stabilized at large negative $R / L_{n}$. There is a region of inward transport at large negative $R / L_{n}$, which however coincides with low growth rates since the TE mode is stabilized in the negative $R / L_{n}$ region. The larger inward particle flux in the ITG case is consistent with a thermodiffusive contribution, which is inward for the ITG mode and outward for the TE mode. $^{21-24}$

In Fig. 3, we compare the eigenvalues of GENE and EDWM in a scan in $R / L_{n}$ with $k_{y} \rho_{s}$ as a parameter. In the mixed case, Fig. 3(a), the real frequencies match well while the growth rates are significantly reduced in GENE for lower and negative $R / L_{n}$. This is expected since the fluid model neglects wave-particle resonances which are usually stabilizing. In the TE dominated case, Fig. 3(b), the growth rates are more similar while the gyrokinetic real frequencies are smaller. As an indication of the direction of the quasilinear gyrokinetic and fluid particle fluxes, $\Gamma /\left(q_{i}+q_{e}\right)$, where $q_{i}$ and $q_{e}$ are the normalized ion and electron heat fluxes, are also shown in Fig. 3. Vertical lines indicate the lowest $R / L_{n}$ where the particle flux changes the sign, an indication of the background peaking factor or $R / L_{n}$ of zero particle flux. Quasilinear simulations have been shown to reproduce well the nonlinear predictions for the particle fluxes. ${ }^{4,25}$ The flux ratios match well for the fluid and gyrokinetic models in the mixed case for lower and negative $R / L_{n}$. The peaking factors also match well in this case with slightly higher values at $k_{y} \rho_{s}=0.3$. In the TE case, the fluid model is much more sensitive to $R / L_{n}$ and $R / L_{n}$ of zero particle flux is lower than that of the gyrokinetic model at $k_{y} \rho_{s}=0.3$ which is the focus of the rest of the linear analysis.

Next, the dependence on $\beta$ is explored in Fig. 4. Plasma $\beta$ effects in GENE are included as magnetic field fluctuations perpendicular to the background field. However, since a concentric circular geometry model is used, there is no Shafranov shift. For negative $R / L_{n}, \beta$ is stabilizing in both models. The MHD ballooning limit can be estimated by $0.6 \hat{s} /\left[q^{2}\left(2 R / L_{n}+R / L_{T_{i}}+R / L_{T_{e}}\right)\right]{ }^{26-29}$ and so, $\beta_{\text {crit, }}$ MHD $=1.8 \%$ for $R / L_{n}=0$ and $\beta_{\text {crit, } \mathrm{MHD}}=6.2 \%$ at $R / L_{n}=-5$. The
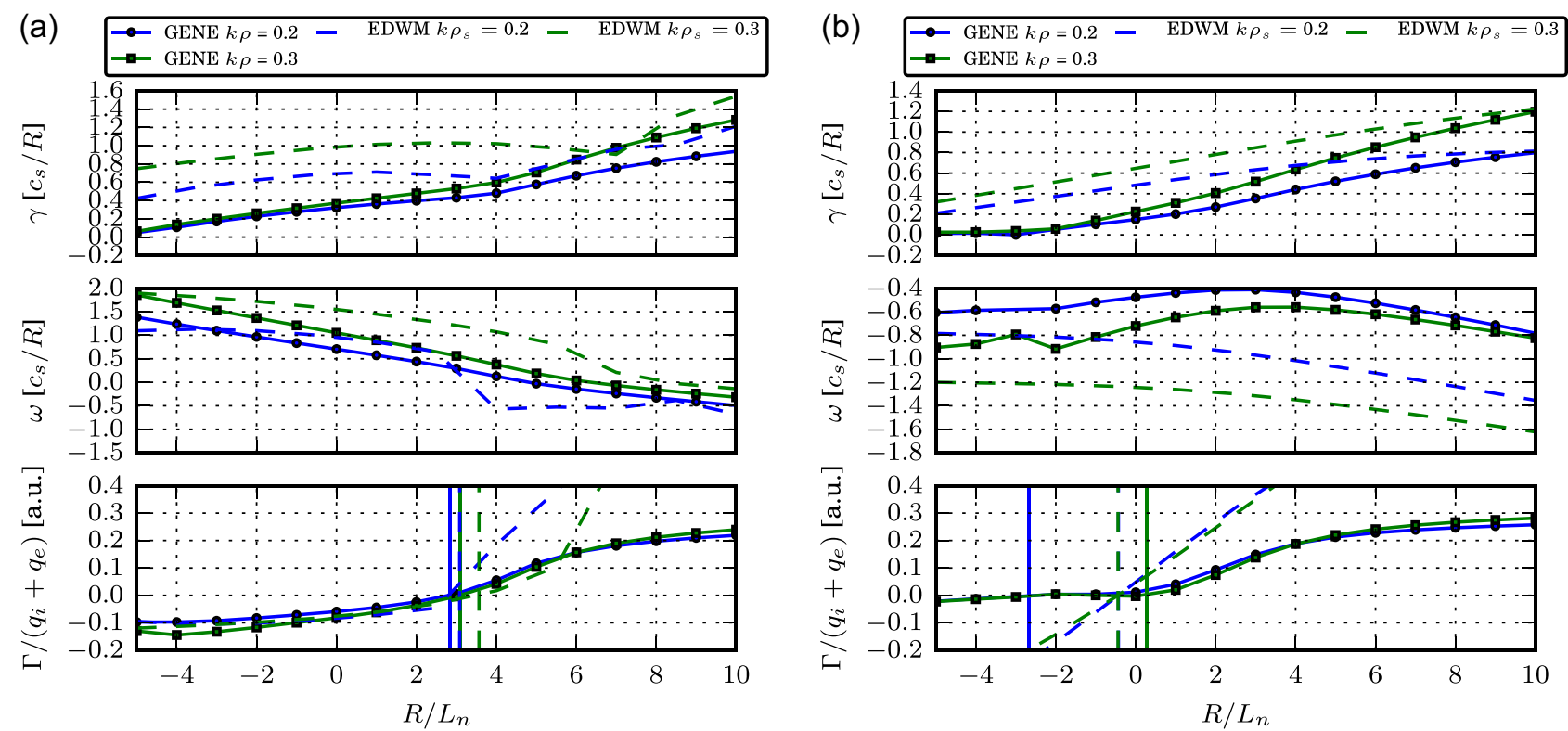

FIG. 3. Comparison of linear gyrokinetic and fluid eigenvalues in the scan over $R / L_{n}$ with $k_{y} \rho_{s}$ as a parameter. Normalized particle flux is also shown. Vertical lines indicate the value of $R / L_{n}$ where the particle flux changes the sign. (a) The mixed ITG/TE case $\left(R / L_{T_{e}}=R / L_{T_{i}}=6.96\right)$. (b) The pure TE case $\left(R / L_{T_{e}}=6.96, R / L_{T_{i}}=0\right)$. 
(a)
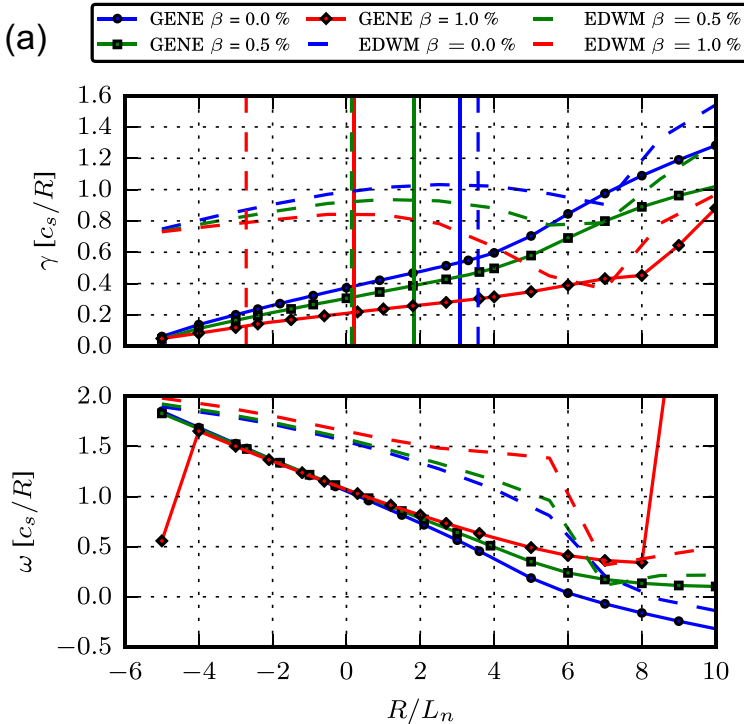

(b)
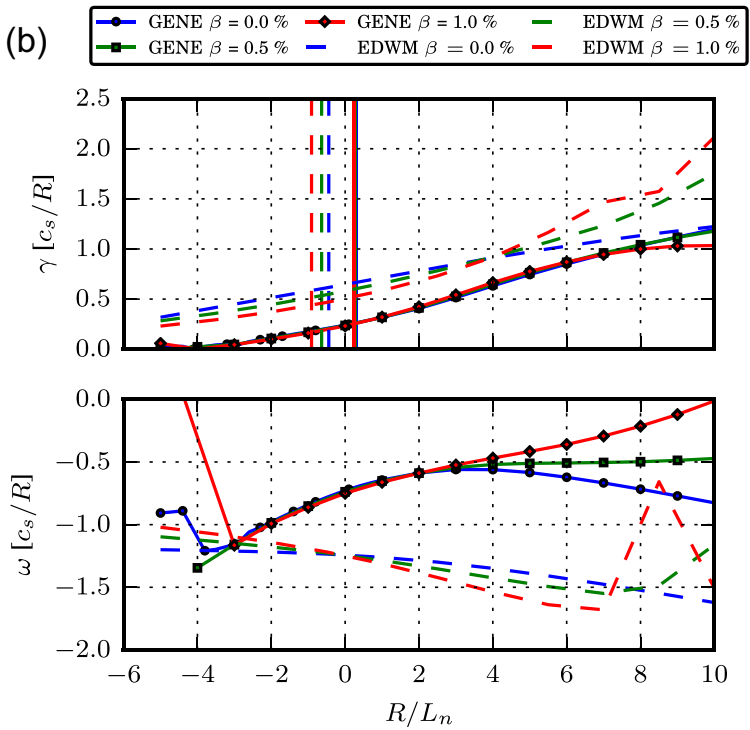

FIG. 4. Linear gyrokinetic and fluid eigenvalues at $k_{y} \rho_{s}=0.3$ in the scan over $R / L_{n}$ with plasma $\beta$ as a parameter. Vertical lines indicate the value of $R / L_{n}$ where the particle flux changes the sign. (a) The mixed ITG/TE case $\left(R / L_{T_{e}}=R / L_{T_{i}}=6.96\right)$. (b) The pure TE case $\left(R / L_{T_{e}}=6.96, R / L_{T_{i}}=0\right)$.

validity of this estimate for negative $R / L_{n}$ has been confirmed with linear gyrokinetic simulations. Thus, for sufficiently large negative $R / L_{n}$, the Kinetic Ballooning Mode (KBM) will not be excited and the ITG mode will be dominant for which $\beta$ has a stabilizing effect, which is consistently found in gyrokinetic simulations. ${ }^{30}$ Both models also show a decreasing peaking factor and flux ratio with increasing $\beta$ in the ITG dominated case, as shown in Fig. 4(a), consistent with earlier gyrokinetic findings. ${ }^{31}$ In the TE dominated case, $\beta$ is stabilizing at small and negative $R / L_{n}$ in the fluid model with reduced peaking factors, while the effect is small in the gyrokinetic model.

In a scaling of collisionality without changing the temperature or density profiles, a larger collisionality does not stabilize the modes significantly in the negative $R / L_{n}$ region in the mixed case in either model, as shown in Fig. 5(a). A reduction in the (still positive) background peaking factor is found, which is a known result. ${ }^{21,31,32}$ The reduction is slightly larger in the fluid model. The effect on the flux ratio for negative $R / L_{n}$ from increased collisionality is small in both models. In the TE case, shown in Fig. 5(b), the stabilization is larger than in the mixed case and this effect is amplified in the fluid model. The effect on the flux ratio is also larger in the fluid model. In Fig. 6, the scaling with magnetic shear is displayed. Larger magnetic shear stabilizes for negative $R / L_{n}$ in both the mixed and TE cases in GENE, while the effect is smaller in EDWM. The positive background peaking factor increases with larger shear in the mixed case. In the TE case, a background peaking factor is not found for the highest values of $\hat{s}$ and otherwise (a)
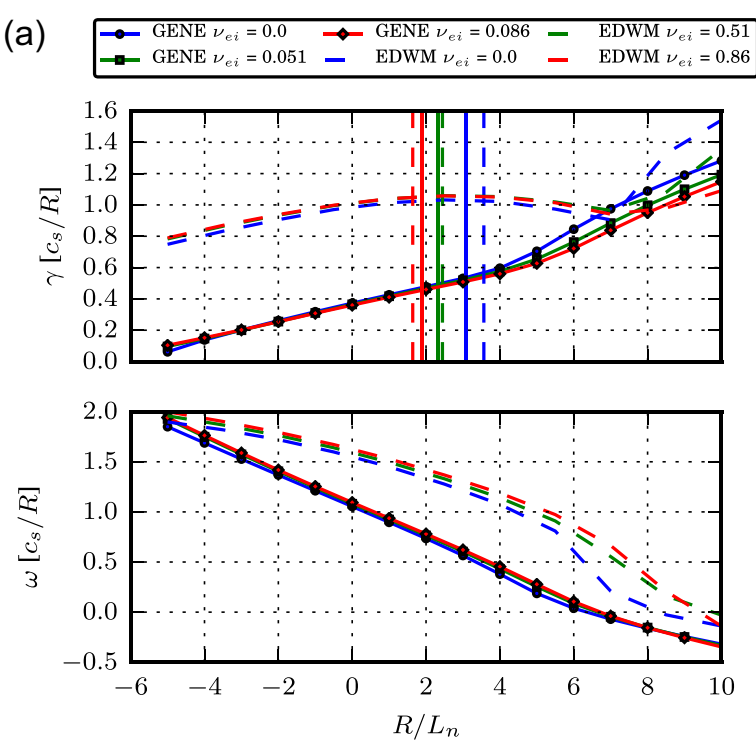

(b)
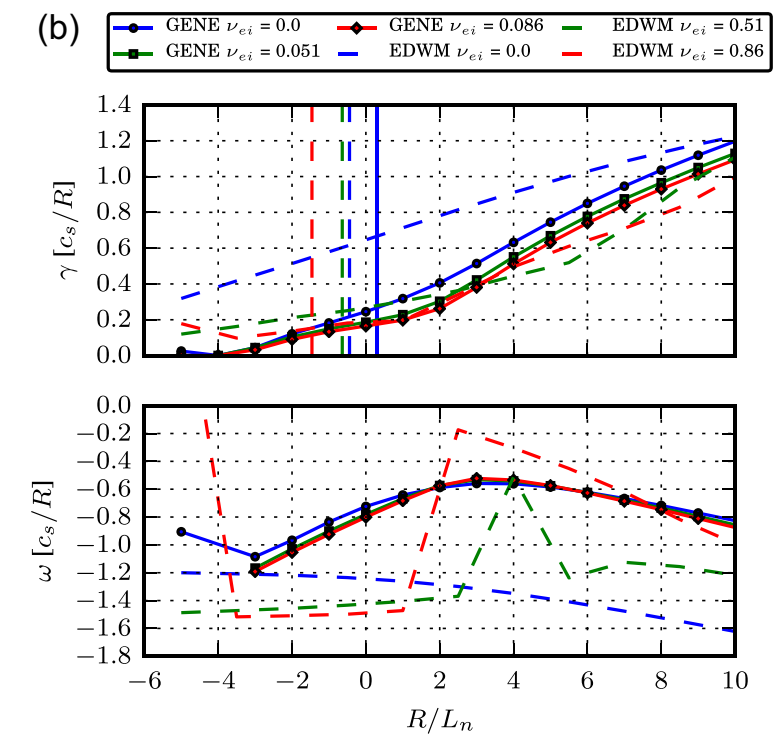

FIG. 5. Linear gyrokinetic and fluid eigenvalues and normalized particle fluxes at $k_{y} \rho_{s}=0.3$ in the scan over $R / L_{n}$ with $\nu_{e i}$, the electron ion collision frequency, as a parameter. Vertical lines indicate the value of $R / L_{n}$ where the particle flux changes the sign. (a) The mixed ITG/TE case $\left(R / L_{T_{e}}=R / L_{T_{i}}=6.96\right)$. (b) The pure TE case $\left(R / L_{T_{e}}=6.96, R / L_{T_{i}}=0\right)$. 
(a)
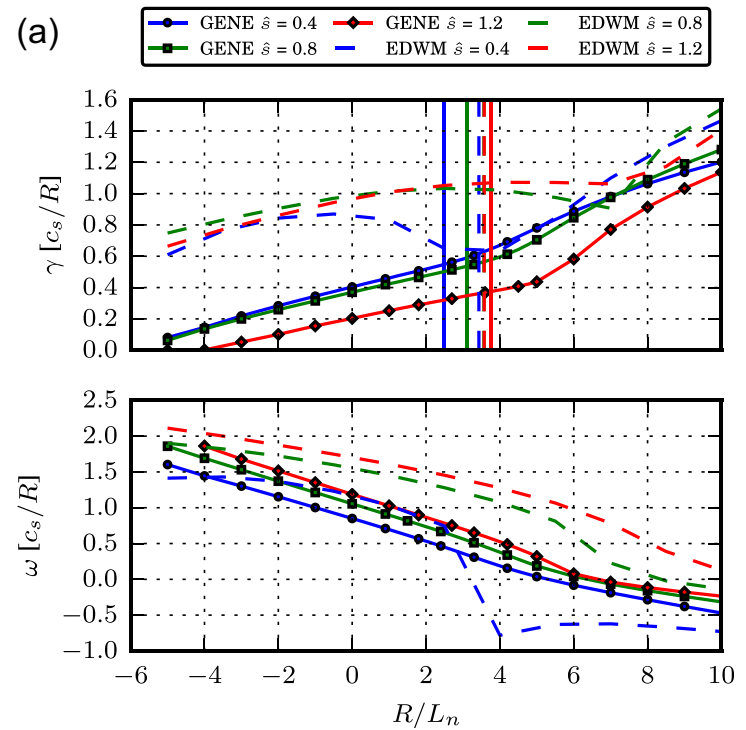

(b)
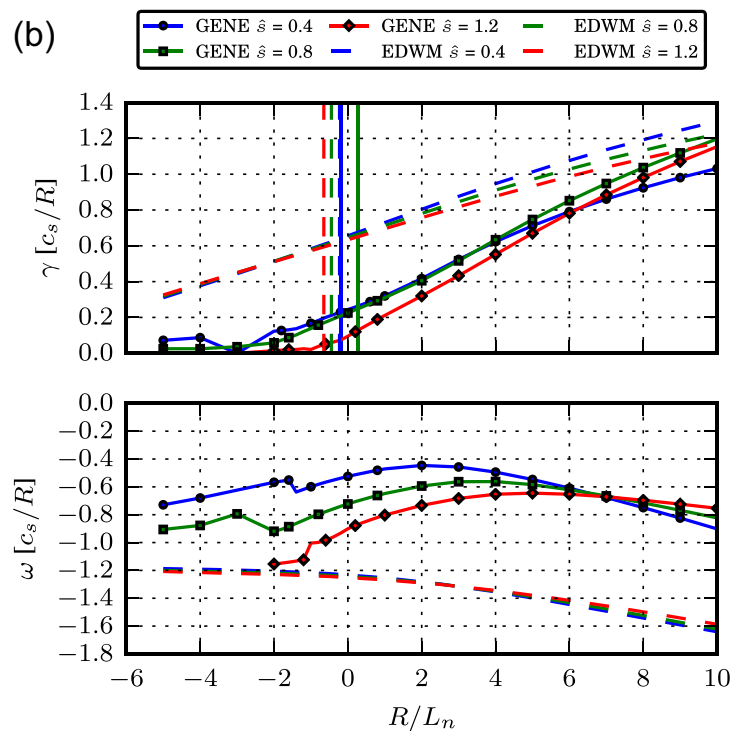

FIG. 6. Linear gyrokinetic and fluid eigenvalues and normalized particle fluxes at $k_{y} \rho_{s}=0.3$ in the scan over $R / L_{n}$ with $\hat{s}$ as a parameter. Vertical lines indicate the value of $R / L_{n}$ where the particle flux changes the sign. (a) The mixed ITG/TE case $\left(R / L_{T_{e}}=R / L_{T_{i}}=6.96\right)$. (b) The pure TE case $\left(R / L_{T_{e}}=6.96, R / L_{T_{i}}=0\right)$.

reduced with lower values of $\hat{s}$ in the gyrokinetic model. In both cases, the effect on the flux ratio for negative $R / L_{n}$ is small. Scans over the safety factor, $q$, and the temperature ratio, $T_{i} / T_{e}$, were also performed. The sensitivity to $q$ was small in both models, while an increase in $T_{i} / T_{e}$ led to a less negative particle flux ratio in the CBC case. In summary, in varying these parameters, the largest sensitivity in the mixed case in the negative $R / L_{n}$ region is to plasma $\beta$, magnetic shear, and $T_{i} / T_{e}$, while in the TE case, the largest sensitivity is to collisionality and magnetic shear. The effect of $\beta$ on the particle transport for negative $R / L_{n}$ is further studied in Sec. IV.

In order to study the instabilities closer to marginal stability, a scan in $R / L_{T}=R / L_{T_{i}}=R / L_{T_{e}}$ and $R / L_{n}$ is shown in Fig. 7. In the negative $R / L_{n}$ region, the ITG mode will eventually be stabilized with decreasing $R / L_{T}$, while for higher $R /$ $L_{n}$, the TE mode turbulence will instead be excited. As shown in Fig. 7(c), this leads to a lower background peaking factor with the decrease in $R / L_{T}$.

\section{NONLINEAR RESULTS}

In order to verify the quasilinear results, nonlinear GENE simulations are needed. For the nonlinear GENE simulations, a simulation domain in the perpendicular plane of $\left[L_{x}, L_{y}\right]=[126,126] \rho_{s}$ is used, with a resolution of $\left[n_{x}\right.$, $\left.n_{y}\right]=[96,48]$. In the parallel direction, 32 grid points are used, and in the parallel velocity direction, 64 grid points and 16 magnetic moments are used. The simulations are typically run up to a simulation time of $t=300 R / c_{s}$, where $R$ is the major radius and $c_{s}=\sqrt{T_{e} / m_{i}}$. The resolution and simulation domain are checked through convergence tests. Nonlinear simulations are performed for the CBC case $\left(R / L_{T_{e}}=R / L_{T_{i}}=6.96\right)$ and compared to the EDWM results at $k_{y} \rho_{s}=0.2$ for different values of plasma $\beta$. The particle fluxes are shown in Fig. 8(a). The fluid and gyrokinetic particle fluxes agree reasonable well at $\beta=0 \%$ and $\beta=0.5 \%$. For $\beta=0 \%$, the particle flux is negative around $R / L_{n}=0$, which means that the particles in a pellet ablation peak would travel

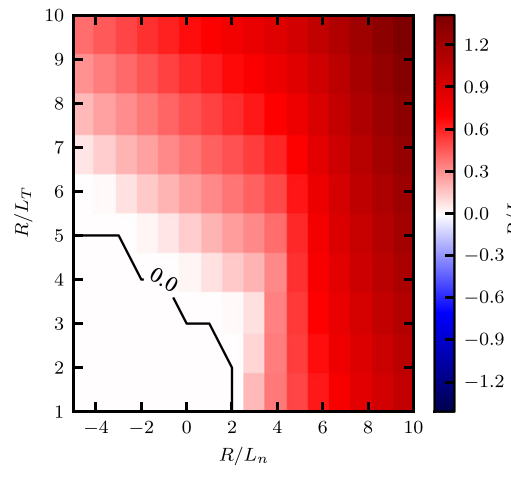

(a)

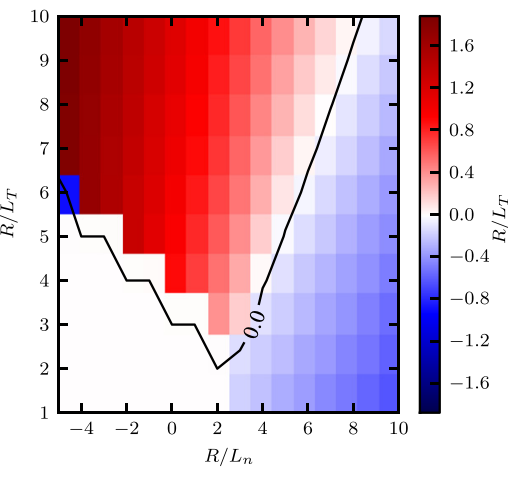

(b)

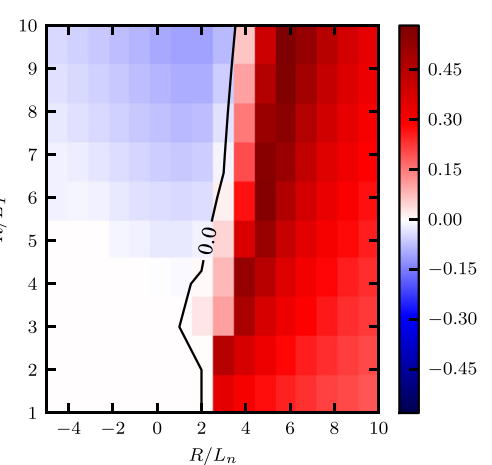

(c)

FIG. 7. GENE eigenvalues as a function of $R / L_{n}$ and $R / L_{T_{i}}=R / L_{T_{e}}$ at $k_{y} \rho_{s}=0.3$. (a) Linear growth rate, $\gamma\left[c_{s} / R\right]$. (b) Linear real frequency, $\omega_{r}\left[c_{s} / R\right]$. (c) Normalized flux, $\Gamma / \delta n^{2}$. 


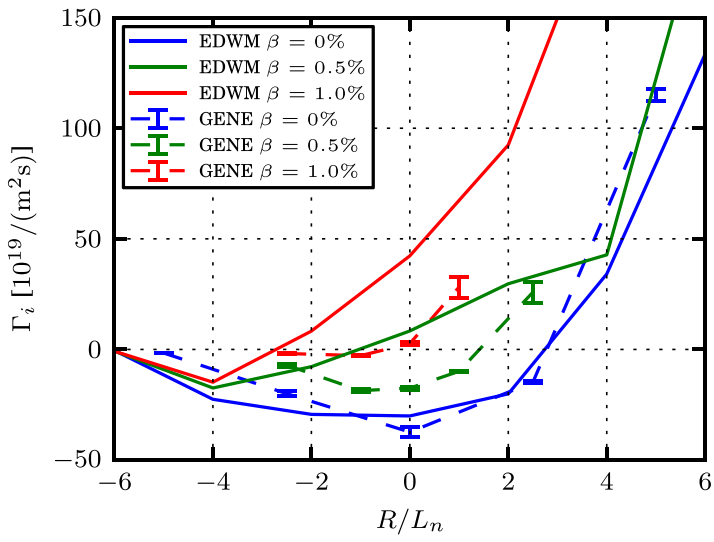

(a)

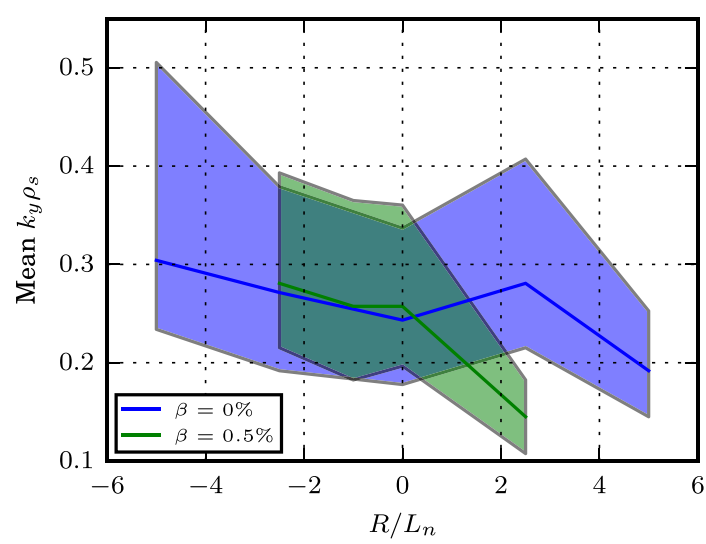

(b)

FIG. 8. Nonlinear results as a function of $R / L_{n}$ and plasma $\beta$. (a) Nonlinear gyrokinetic particle flux compared to the EDWM results. (b) Mean $k_{y} \rho_{s}$ of the ion particle flux.

inwards. However, at higher $\beta$, the transport decreases in the negative $R / L_{n}$ region, while it changes sign to outward in the positive region. In this parameter regime, the pellet fuelling scheme would be less efficient. In Fig. 8(b), the mean $k_{y} \rho_{s}$ for the simulations is shown. The shaded region represents the wave numbers responsible for $25 \%$ of the flux over and under the mean and thus indicates the width of the particle flux spectra. The mean $k_{y} \rho_{s}$ as well as the width of the particle spectra increases when going to a more negative $R / L_{n}$.

\section{EFFECTS OF FAST PARTICLES}

Fast ions have been shown to stabilize the ITG mode linearly and nonlinearly through dilution of the main ion species, ${ }^{33,34}$ Shrafranov shift stabilization, ${ }^{35}$ and electromagnetic stabilization through the suprathermal pressure gradients. ${ }^{36}$ Nonlinearly, it has been shown that it is the last of these effects that dominates. ${ }^{18,37}$ This electromagnetic effect has been shown to be more effective at low magnetic shear, ${ }^{18}$ but the analysis is here restricted to $\hat{s}=0.8$ since pellet fuelling typically affects the profiles at larger radii where the shear is larger. The geometric stabilization whereby the increased Shafranov shift leads to stabilization is not modelled here. In the present modelling, the fast ions are added as a species with a Maxwellian background distribution. ${ }^{38}$ Following Ref. 39, a temperature of $T_{\text {fast }}=35 \mathrm{keV}$, $R / L_{T_{\text {fast }}}=0$, and $n_{\text {fast }}=0.1 n_{e}$ are chosen. The fast particle simulations are run without collisions and typically with a $\beta_{e}$ of $0.5 \%$. Because of the quasineutrality condition on the gradients, $\sum_{j} n_{j} q_{j} \frac{R}{L_{n_{j}}}=0$, imposed in GENE, the background density gradient of either the ions or the electrons must be adapted if adding a fast particle population. Hence, the combined effect of fast particles and the change in background density gradients is explored in Fig. 9 where the linear eigenvalues are shown together with the density gradient of zero quasi-linear particle flux. In 9 (a), $R / L_{n_{i}}$ is modified for a given electron and fast particle density gradient, resulting in a larger stabilization than if $R / L_{n_{e}}$ is modified, as shown in Fig. 9(b). This indicates that the modification of the ion density gradient is the cause of the significant linear stabilization of the ITG mode in the positive gradient region. However, in the following, we will focus on the more realistic case where

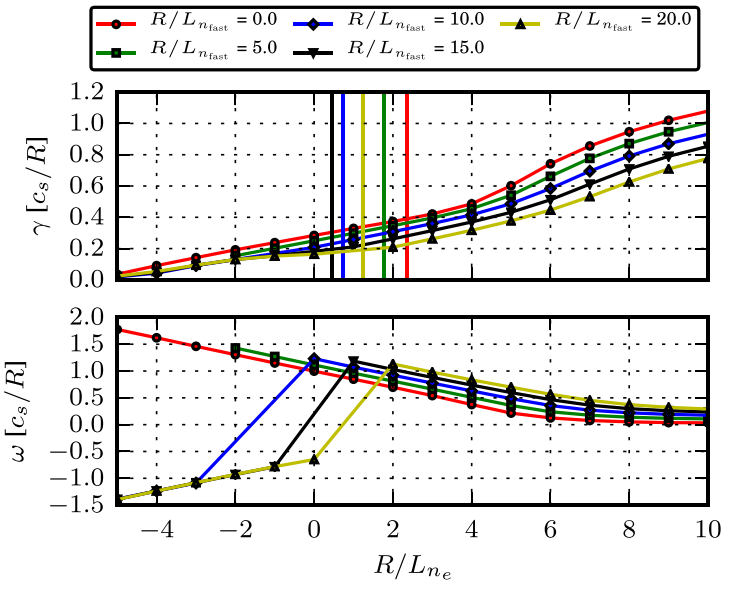

(a)

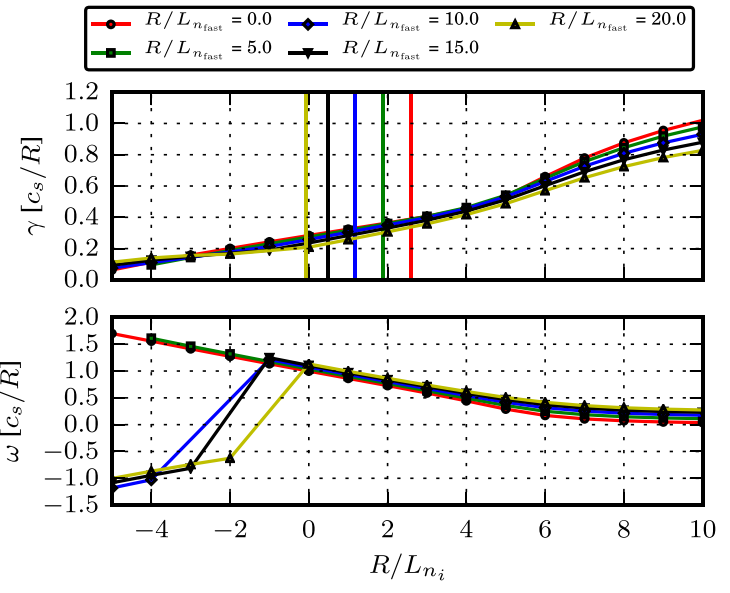

(b)

FIG. 9. Linear gyrokinetic eigenvalues at $k_{y} \rho_{s}=0.3$, indicating the linear stabilization with the fast particle density gradient in the cases where either $R / L_{n_{e}}$ or $R / L_{n_{i}}$ is kept fixed. Vertical lines indicate the value of $R / L_{n_{i}}$ where the particle flux changes the sign. (a) $R / L_{n_{e}}$ kept fixed; the fast particle density gradient modifies $R / L_{n_{i}}$. (b) $R / L_{n_{i}}$ kept fixed; the fast particle density gradient modifies $R / L_{n_{e}}$. 


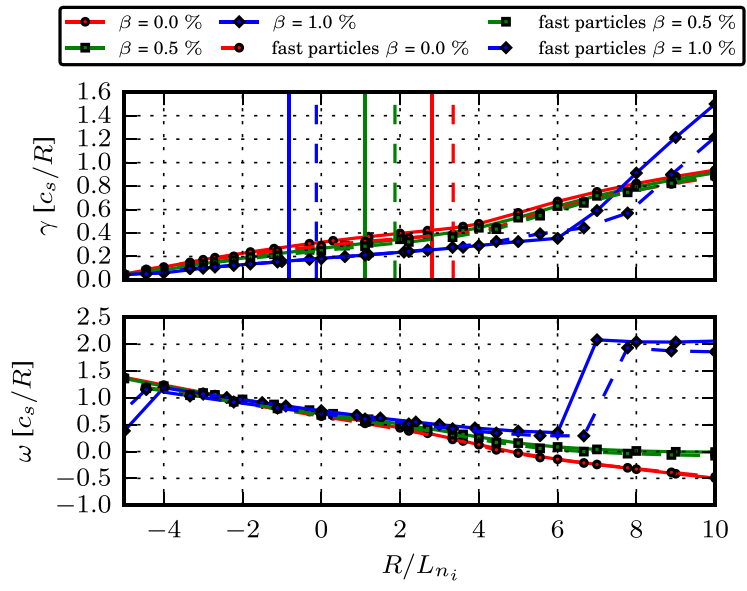

(a)

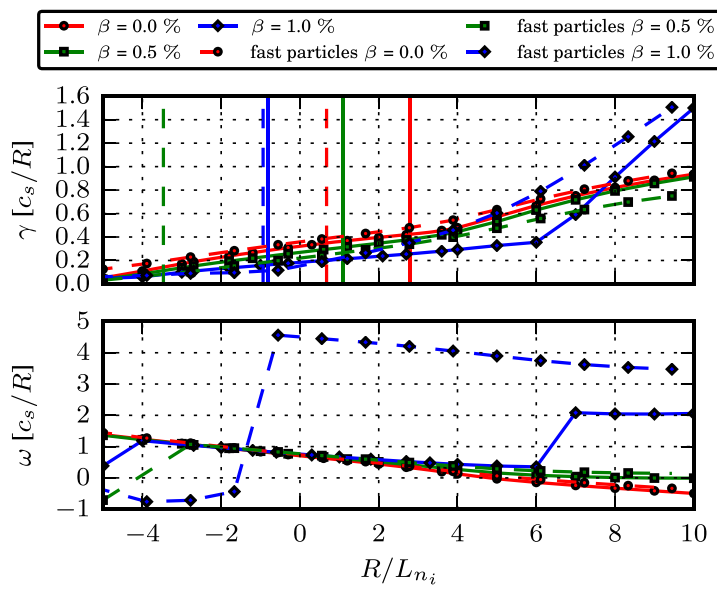

(b)

FIG. 10. Linear gyrokinetic eigenvalues at $k_{y} \rho_{s}=0.2$ in the scan over $R / L_{n_{i}}$ with plasma $\beta$ as a parameter with fast particles with $R / L_{n_{\text {fast }}}=0$ or $R / L_{n_{\text {fast }}}=15$. Vertical lines indicate the value of $R / L_{n_{i}}$ where the particle flux changes the sign. (a) $R / L_{n_{\text {fast }}}=0$. (b) $R / L_{n_{\text {fast }}}=15$.

the electron density gradient is modified. The background peaking factor is generally reduced with increasing $R / L_{n_{\mathrm{fass}}}$, with the exception of larger values of $R / L_{n_{\text {fast }}}$ in the case where the fast particle gradient modifies $R / L_{n_{i}}$ where the TE mode becomes dominant, increasing the peaking factor.

In Fig. 10, the effect from the fast particles is shown versus $R / L_{n_{i}}$ with $\beta$ as a parameter for the cases $R / L_{n_{\text {fast }}}=0$ and $R / L_{n_{\text {fast }}}=15$. In the first case, the resulting growth rates are similar and there is a slight stabilization with fast particles at $\beta=0$. The resulting ion density gradient of zero particle flux (peaking factors) is increased compared to the case without fast particles. In the second case, fast particles and non-zero $\beta$, combined with the adaption of $R / L_{n_{e}}$, instead serve to stabilize the ITG mode for low and negative $R / L_{n_{i}}$, resulting in TE mode turbulence becoming dominant at higher $\beta$ and negative $R / L_{n_{i}}$. The background peaking factor is in the ITG dominated cases, here reduced when adding fast particles.

In order to investigate the fast particle effects on particle transport, nonlinear simulations with fast particles are performed for six different cases as defined in Table II. The three main cases are case 1 with a flat fast particle density profile, case 2 with $R / L_{n_{\text {fast }}}=15$ and $R / L_{n_{i}}=-2.5$, and case 3 with $R / L_{n_{\text {fast }}}=15$ and $R / L_{n_{e}}=-2.5$ resulting in $R / L_{n_{i}}=-4.44$. All are run with $\beta_{e}=0.5 \%$ and without collisions. In order to quantify the effect of fast particles, these cases are compared with cases where the temperature of the fast particle species is reduced to that of the background ions, $T_{\text {fast }}=T_{i}=2.85 \mathrm{keV}$. First, the linear eigenvalue spectra of the three main cases are shown in Fig. 11. The ITG

TABLE II. Nonlinear simulations with fast particles.

\begin{tabular}{lcccc}
\hline \hline Case & $R / L_{n_{\text {fast }}}$ & $R / L_{n_{i}}$ & $R / L_{n_{e}}$ & $T_{\text {fast }}(\mathrm{keV})$ \\
\hline Case 1 & 0 & -2.78 & -2.5 & 35 \\
Case 2 & 15 & -2.5 & -0.75 & 35 \\
Case 3 & 15 & -4.44 & -2.5 & 35 \\
Case 1b & 0 & -2.78 & -2.5 & 2.85 \\
Case 2b & 15 & -2.5 & -0.75 & 2.85 \\
Case 3b & 15 & -4.44 & -2.5 & 2.85 \\
\hline \hline
\end{tabular}

growth rates are similar in case 1 and the case without fast particles. In case 2, the ITG growth rate is significantly reduced, and in case 3, the ITG mode is stabilized, leaving the TE mode as the most unstable for all $k_{y} \rho_{s}$. This is consistent with the ITG stabilization being from the pressure gradient. A dimensionless measure of the pressure gradient is $\alpha \equiv q^{2} \sum_{j} \beta_{j}\left(R / L_{n_{j}}+R / L_{T_{j}}\right)$, where the sum is over all species. ${ }^{18}$ This parameter is a factor $\sim 3$ larger in cases 2 and 3 than in the case without fast particles and case 1. Scans in $T_{\text {fast }}$ for the three cases are shown in Fig. 12. For case 1, shown in Fig. 12(a), higher $T_{\text {fast }}$ slightly destabilizes the ITG mode, and for cases 2 and 3, shown in Figs. 12(b) and 12(c), higher $T_{\text {fast }}$ destabilizes the ITG mode turbulence to a point, but at the very high $T_{\text {fast }}$, corresponding to the nonlinear cases, the ITG mode is again stabilized compared to the $T_{\text {fast }}$ $=T_{i}$ case.

The particle and heat diffusivities from the nonlinear simulations are shown in Fig. 13. In case 1, the diffusivities are similar to the case without fast particles, in agreement with the linear analysis. In case 2, the particle diffusion coefficients and ion heat diffusivities are reduced compared to the case without fast particles and compared to case 1, following the linear results. In case 3 , the particle diffusivities

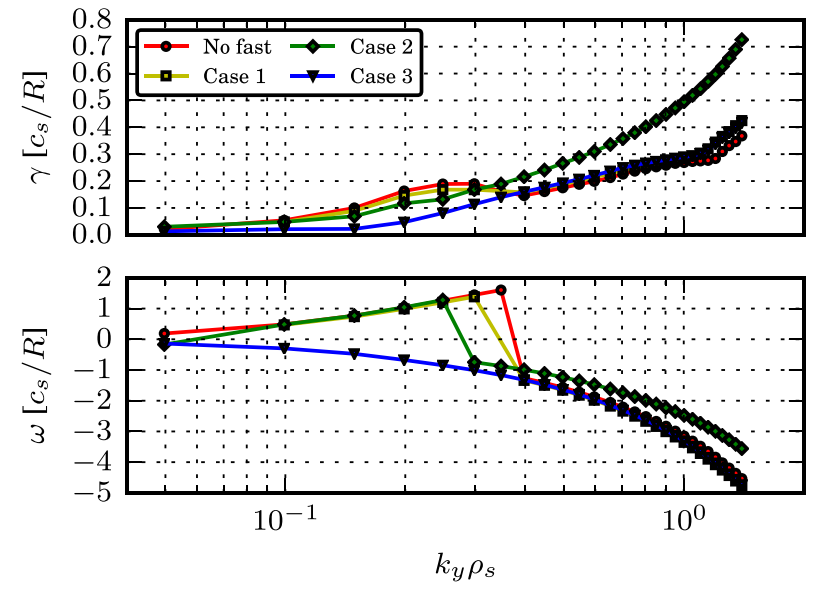

FIG. 11. Linear eigenvalues of the three main cases. 

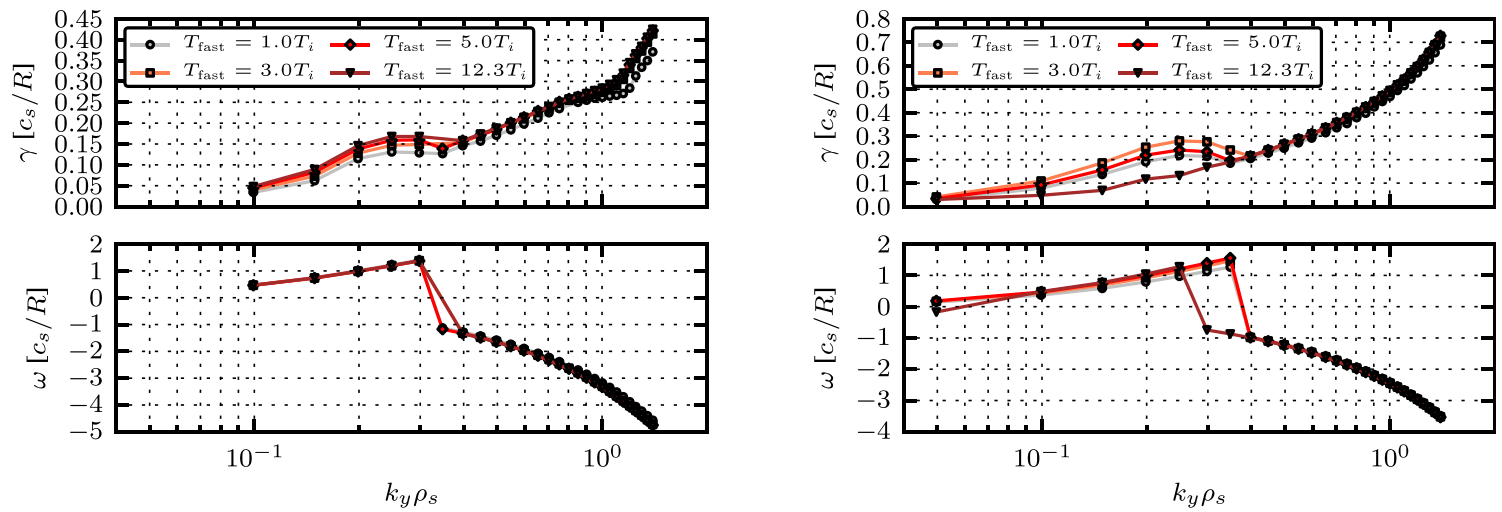

(a)

(b)
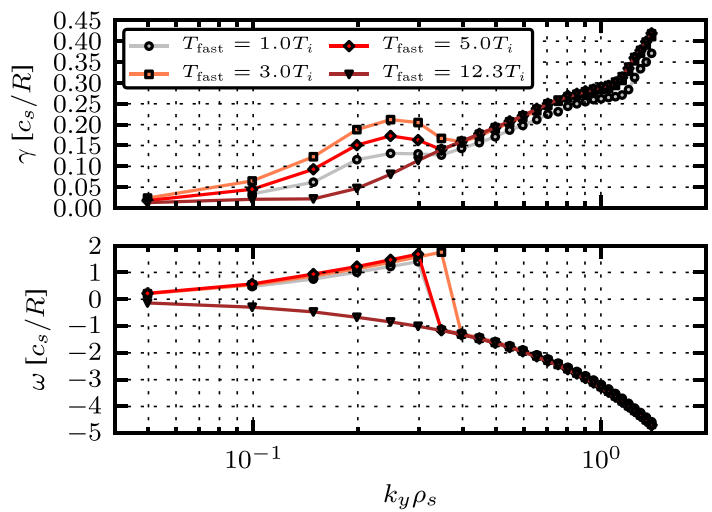

(c)

FIG. 12. Linear eigenvalue spectra in the scan over $T_{\text {fast }}$. (a) Case 1. (b) Case 2. (c) Case 3.

and the ion heat diffusivity are reduced further and the heat transport is dominated by the electron channel, as a result of the stabilization of the ITG mode that was found in the linear analysis for this case.

Turning now to the comparison with the cases with $T_{\text {fast }}$ $=T_{i}=2.85 \mathrm{keV}$, for case $1 \mathrm{~b}$ with $R / L_{n_{\text {fast }}}=0$, the lower $T_{\text {fast }}$ which resulted in reduced linear growth rates also results in reduced diffusivities. For cases $2 \mathrm{~b}$ and $3 \mathrm{~b}$ with $R / L_{n_{\text {fast }}}=15$, on the other hand, the reduced $T_{\text {fast }}$ results in larger diffusivities, in agreement with the stabilization seen in the linear results for the highest fast particle temperatures. In summary, the addition of fast particles with sufficiently high $T_{\text {fast }}$ can

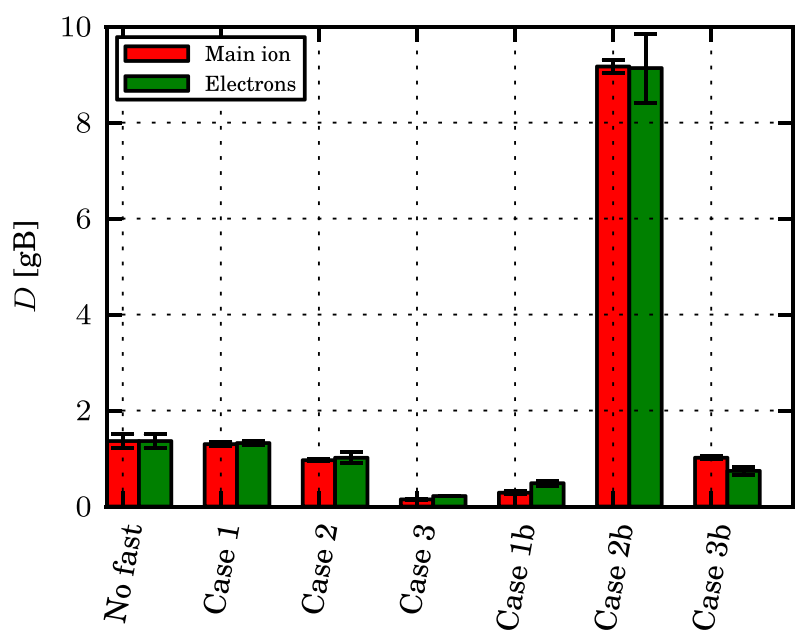

(a)

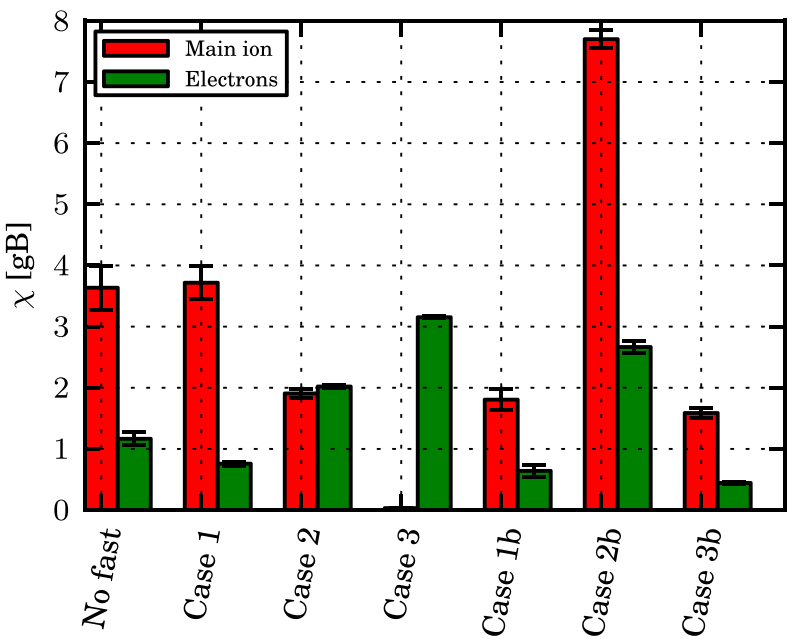

(b)

FIG. 13. Nonlinear diffusion coefficients and heat diffusivities, simulations including fast particles for the cases defined in Table II. (a) Effective diffusion coefficients. (b) Effective heat diffusivities. 


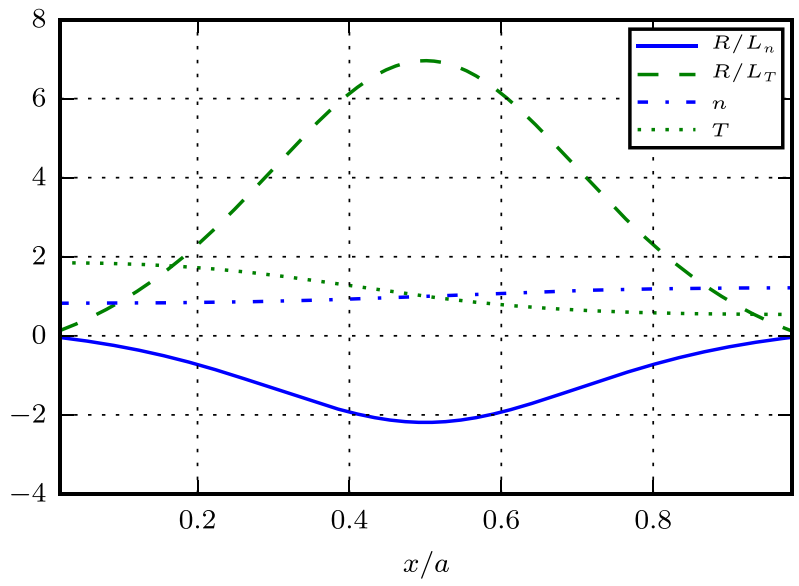

FIG. 14. Radial profiles of temperature and density and their logarithmic gradients for a global simulation.

further serve to decrease the inward particle flux in the positive gradient region.

\section{NONLOCAL EFFECTS}

As $R / L_{n}$ for a hollow density profile can easily vary over a length shorter than several hundred ion gyroradii, it is also worthwhile to investigate the role of nonlocal effects. For this purpose, we perform several global simulations with $\rho_{*}=\rho_{i} / a=1 / 300$. Due to the higher computational cost of global simulations, we restrict the study to electrostatic effects and adiabatic electrons. The physical parameters at the position $x / a=0.5$ correspond to the local simulations described in Table I, and we again use circular concentric flux-surfaces, where the $q$-profile is monotonically increasing. The profiles of the logarithmic gradients of temperature and density are chosen as a peaked Gaussian-like shape (Fig. 14) where the peak amplitude at $x / a=0.5$ equals the value picked in local simulations.

The simulation domain used is $\left[L_{x}, L_{y}\right]=[290,331] \rho_{i}$ in the perpendicular direction with a resolution of $\left[n_{x}, n_{k_{y}}\right]$

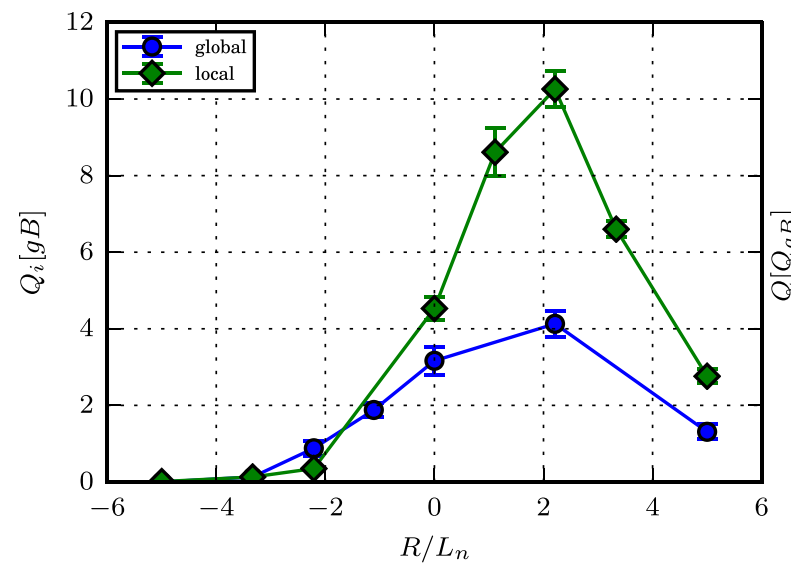

(a)
$=[384,48]$. Simulations are run up to $t=1000 a / c_{s}$ for optimal statistical analysis in this case.

In Fig. 15(a), the average turbulent heat flux in the region $x / a=0.4-0.6$ is compared with local adiabatic electron simulations (with $R / L_{n}$ corresponding to the peak of the global profile). For positive $R / L_{n}$, the difference between local and global simulations reproduces the well-established observation that turbulent transport is smaller for finite $\rho_{*}{ }^{40-43}$ due to a different structure of global modes. ${ }^{44-46}$ The dependence on $R / L_{n}$ remains qualitatively similar. The results for negative $R / L_{n}$, however, suggest that turbulence is less affected by negative $R / L_{n}$ in the global than in the fluxtube case. This is also clearly visible in the time-averaged heat flux profile for $R / L_{n}(x=0.5 a)=-2.2$ where the global simulation produces consistently more flux in the central region.

This result is an indication that nonlocal effects can also play a subtle role in transport in configurations with hollow density profiles and we plan to further investigate such systems with a small negative $R / L_{n}$.

\section{CONCLUSION}

In this paper, the characteristics of particle transport driven by ITG/TE mode turbulence in regions of hollow density profiles were studied by fluid as well as gyrokinetic simulations, using the EDWM and GENE codes in parameter scans around those of the Cyclone Base Case. In the linear analysis, two main cases were studied, the original Cyclone Base Case with $R / L_{T_{e}}=R / L_{T_{i}}=6.96$ where both the ITG and TE modes were excited, a case with $R / L_{T_{i}}=6.96$ and $R / L_{T_{e}}=0$ where mainly the ITG mode was driven unstable. In the linear gyrokinetic analysis of the mixed case, it was found that the ITG mode is dominant in the negative $R / L_{n}$ region for $k_{y} \rho_{s}<0.5$ and that the TE mode was dominant otherwise, with a background peaking factor between $P F \approx 3$ at $k_{y} \rho_{s}=0.2$ and 6 at $k_{y} \rho_{s}=1.2$. Plasma $\beta$ was found to have a stabilizing effect in the mixed and ITG cases in the negative $R / L_{n}$ region in both GENE and EDWM. Both models indicated a decrease in the background peaking factor with

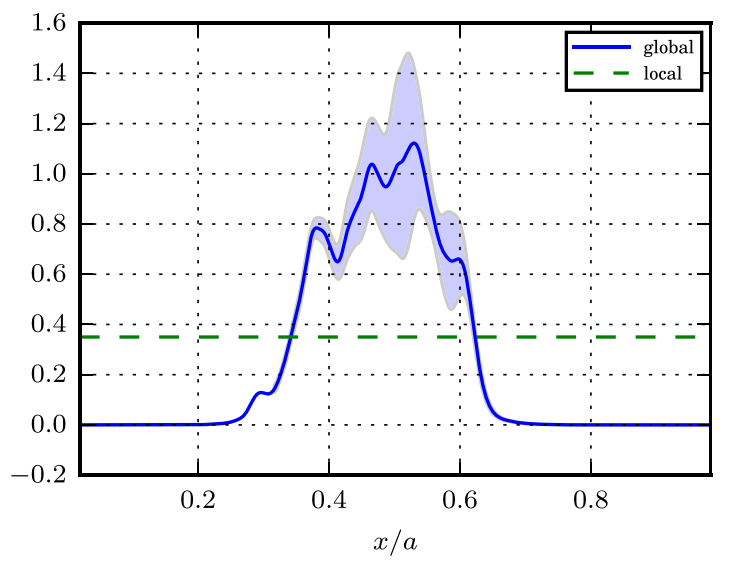

(b)

FIG. 15. Comparison of time-averaged radial heat fluxes between local and global $\left(\rho_{*}=1 / 300\right)$ nonlinear simulations. (a) Nonlinear gyrokinetic heat flux as a function of $R / L_{n}$. (b) Radial profile for $R / L_{n}=-2.2$. 
increased plasma $\beta$ in the mixed case. Increasing magnetic shear also had a stabilizing effect in the negative $R / L_{n}$ region in GENE, while the effect is smaller in EDWM with little effect on the flux ratio in both models. Adding collisions lowered the positive peaking factor in the mixed case, while it had a negligible effect on the growth rates. In the nonlinear analysis, the particle fluxes are negative around $R / L_{n}=0.0$ in the electrostatic case. Adding $\beta$ effects, however, the inward particle flux in the negative $R / L_{n}$ region decreases in both models, while it changes the sign to outwards in this region with a sufficiently high $\beta$. This agreed with the linear results which indicated a decrease in the background peaking factor. Adding fast particles might further decrease the inward particle flux in the negative $R / L_{n}$ region. This may have serious consequences for the efficiency of the pellet fuelling scheme in high $\beta$ plasmas. An initial global study of the heat flux in ITG turbulence with adiabatic electrons demonstrated that nonlocal effects can play a subtly different role from usual in a hollow density profile, motivating further studies.

\section{ACKNOWLEDGMENTS}

The simulations were performed on resources provided by the Swedish National Infrastructure for Computing (SNIC) at the PDC Centre for High Performance Computing (PDC-HPC), on the HELIOS supercomputer system at the Computational Simulation Centre of International Fusion Energy Research Centre (IFERC-CSC), Aomori, Japan, under the Broader Approach collaboration between Euratom and Japan, implemented by Fusion for Energy and JAEA and at the Marconi supercomputer system, at Cineca, Casalecchio di Reno, Italy. This work was funded by a grant from The Swedish Research Council (C0338001).

${ }^{1}$ L. Garzotti, J. Figueiredo, C. M. Roach, M. Valovič, D. Dickinson, G. Naylor, M. Romanelli, R. Scannell, and G. Szepesi, "Microstability analysis of pellet fuelled discharges in MAST," Plasma Phys. Controlled Fusion 56(3), 035004 (2014).

${ }^{2}$ M. Kotschenreuther, G. Rewoldt, and W. M. Tang, "Comparison of initial value and eigenvalue codes for kinetic toroidal plasma instabilities," Comput. Phys. Commun. 88(2), 128-140 (1995).

${ }^{3}$ D. Tegnered, H. Nordman, M. Oberparleiter, P. Strand, L. Garzotti, I. Lupelli, C. M. Roach, M. Romanelli, and M. Valovic, "Gyrokinetic simulations of transport in pellet fuelled discharges at JET," in 43rd European Physical Society Conference on Plasma Physics, Leuven, Belgium, 2016.

${ }^{4}$ C. Bourdelle, X. Garbet, F. Imbeaux, A. Casati, N. Dubuit, R. Guirlet, and T. Parisot, "A new gyrokinetic quasilinear transport model applied to particle transport in tokamak plasmas," Phys. Plasmas 14(11), 112501 (2007). ${ }^{5}$ B. Baiocchi, C. Bourdelle, C. Angioni, F. Imbeaux, A. Loarte, and M. Maslov, "Transport analysis and modelling of the evolution of hollow density profiles plasmas in JET and implication for ITER," Nucl. Fusion 55(12), 123001 (2015).

${ }^{6}$ L. Garzotti, X. Garbet, P. Mantica, V. Parail, M. Valovič, G. Corrigan, D. Heading, T. T. C. Jones, P. Lang, H. Nordman, B. Pégourié, G. Saibene, J. Spence, P. Strand, J. Weiland, and Contributors to the EFDA-JET Workprogramme, "Particle transport and density profile analysis of different jet plasmas," Nucl. Fusion 43(12), 1829 (2003).

${ }^{7}$ See http://genecode.org/ for further information about the GENE code.

${ }^{8}$ B. B. Kadomtsev and O. P. Pogutse, "Trapped particles in toroidal magnetic systems," Nucl. Fusion 11(1), 67 (1971).

${ }^{9}$ B. Coppi and F. Pegoraro, "Theory of the ubiquitous mode," Nucl. Fusion 17(5), 969 (1977).

${ }^{10}$ W. Horton, Jr., D. Choi, and W. M. Tang, "Toroidal drift modes driven by ion pressure gradients," Phys. Fluids 24(6), 1077-1085 (1981).
${ }^{11}$ P. N. Guzdar, L. Chen, W. M. Tang, and P. H. Rutherford, "Ion temperature gradient instability in toroidal plasmas," Phys. Fluids 26(3), 673-677 (1983).

${ }^{12} \mathrm{~F}$. Romanelli, "Ion temperature-gradient-driven modes and anomalous ion transport in tokamaks," Phys. Fluids B: Plasma Phys. 1(5), 1018-1025 (1989).

${ }^{13}$ H. Biglari, P. H. Diamond, and M. N. Rosenbluth, "Toroidal ion-pressuregradient-driven drift instabilities and transport revisited," Phys. Fluids B: Plasma Phys. 1(1), 109-118 (1989).

${ }^{14}$ T. Dannert and F. Jenko, "Gyrokinetic simulation of collisionless trappedelectron mode turbulence," Phys. Plasmas 12(7), 072309 (2005).

${ }^{15}$ A. M. Dimits, G. Bateman, M. A. Beer, B. I. Cohen, W. Dorland, G. W. Hammett, C. Kim, J. E. Kinsey, M. Kotschenreuther, A. H. Kritz et al., "Comparisons and physics basis of tokamak transport models and turbulence simulations," Phys. Plasmas 7(3), 969-983 (2000).

${ }^{16} \mathrm{~J}$. Weiland, Collective Modes in Inhomogeneous Plasmas: Kinetic and Advanced Fluid Theory (CRC Press, 1999).

${ }^{17}$ P. I. Strand, G. Bateman, A. Eriksson, W. A. Houlberg, A. H. Kritz, H. Nordman, and J. Weiland, "Comparisons of anomalous and neoclassical contributions to core particle transport in tokamak discharges," in 31th EPS Conference, London 2004, European Physical Society (2004), Vol. 28.

${ }^{18}$ J. Citrin, F. Jenko, P. Mantica, D. Told, C. Bourdelle, J. Garcia, J. W. Haverkort, G. M. D. Hogeweij, T. Johnson, and M. J. Pueschel, "Nonlinear stabilization of tokamak microturbulence by fast ions," Phys. Rev. Lett. 111(15), 155001 (2013).

${ }^{19}$ F. Merz, "Gyrokinetic simulation of multimode plasma turbulence," Ph.D. thesis (Universitat Munster, 2008).

${ }^{20}$ H. Nordman, A. Skyman, P. Strand, C. Giroud, F. Jenko, F. Merz, V. Naulin, T. Tala, and JET-EFDA Contributors, "Fluid and gyrokinetic simulations of impurity transport at JET," Plasma Phys. Controlled Fusion 53(10), 105005 (2011).

${ }^{21}$ ASDEX Upgrade Team, C. Angioni, A. G. Peeters, G. V. Pereverzev, F. Ryter, and G. Tardini, "Density peaking, anomalous pinch, and collisionality in tokamak plasmas," Phys. Rev. Lett. 90(20), 205003 (2003).

${ }^{22}$ C. Angioni and A. G. Peeters, "Direction of impurity pinch and auxiliary heating in tokamak plasmas," Phys. Rev. Lett. 96(9), 095003 (2006).

${ }^{23}$ X. Garbet, L. Garzotti, P. Mantica, H. Nordman, M. Valovic, H. Weisen, and C. Angioni, "Turbulent particle transport in magnetized plasmas," Phys. Rev. Lett. 91(3), 035001 (2003).

${ }^{24}$ C. Angioni, E. Fable, M. Greenwald, M. Maslov, A. G. Peeters, H. Takenaga, and H. Weisen, "Particle transport in tokamak plasmas, theory and experiment," Plasma Phys. Controlled Fusion 51(12), 124017 (2009).

${ }^{25}$ A. Casati, C. Bourdelle, X. Garbet, F. Imbeaux, J. Candy, F. Clairet, G. Dif-Pradalier, G. Falchetto, T. Gerbaud, V. Grandgirard et al., "Validating a quasi-linear transport model versus nonlinear simulations," Nucl. Fusion 49(8), 085012 (2009).

${ }^{26}$ M. J. Pueschel, M. Kammerer, and F. Jenko, "Gyrokinetic turbulence simulations at high plasma beta," Phys. Plasmas 15, 102310 (2008).

${ }^{27}$ J. W. Connor, R. J. Hastie, and J. B. Taylor, "Shear, periodicity, and plasma ballooning modes," Phys. Rev. Lett. 40(6), 396 (1978).

${ }^{28}$ B. Coppi, "Topology of ballooning modes," Phys. Rev. Lett. 39(15), 939 (1977).

${ }^{29}$ A. M. M. Todd, M. S. Chance, J. M. Greene, R. C. Grimm, J. L. Johnson, and J. Manickam, "Stability limitations on high-beta tokamaks," Phys. Rev. Lett. 38(15), 826 (1977).

${ }^{30}$ F. Jenko and W. Dorland, "Nonlinear electromagnetic gyrokinetic simulations of tokamak plasmas," Plasma Phys. Controlled Fusion 43(12A), A141 (2001).

${ }^{31}$ A. Skyman, D. Tegnered, H. Nordman, and P. Strand, "Gyrokinetic modelling of stationary electron and impurity profiles in tokamaks," Phys. Plasmas 21(9), 092305 (2014)

${ }^{32}$ C. Angioni, J. Candy, M. Fable, E. Maslov, A. G. Peeters, R. E. Waltz, and H. Weisen, "Particle pinch and collisionality in gyrokinetic simulations of tokamak plasma turbulence," Phys. Plasmas 16(6), 060702 (2009).

${ }^{33}$ C. Holland, L. Schmitz, T. L. Rhodes, W. A. Peebles, J. C. Hillesheim, G. Wang, L. Zeng, E. J. Doyle, S. P. Smith, R. Prater et al., "Advances in validating gyrokinetic turbulence models against L-and H-mode plasmas," Phys. Plasmas 18(5), 056113 (2011).

${ }^{34}$ G. Tardini, J. Hobirk, V. G. Igochine, C. F. Maggi, P. Martin, D. McCune, A. G. Peeters, A. C. C. Sips, A. Stäbler, J. Stober, and ASDEX Upgrade Team. "Thermal ions dilution and ITG suppression in ASDEX upgrade ion ITBS," Nucl. Fusion 47(4), 280 (2007).

${ }^{35}$ C. Bourdelle, G. T. Hoang, X. Litaudon, C. M. Roach, T. Tala, ITPA Topical Group on Transport, ITB Physics, and International ITB Database 
Working Group, "Impact of the $\alpha$ parameter on the microstability of internal transport barriers," Nucl. Fusion 45(2), 110 (2005).

${ }^{36} \mathrm{M}$. Romanelli, A. Zocco, and F. Crisanti, and JET-EFDA Contributors, "Fast ion stabilization of the ion temperature gradient driven modes in the joint European torus hybrid-scenario plasmas: A trigger mechanism for internal transport barrier formation," Plasma Phys. Controlled Fusion 52(4), 045007 (2010).

${ }^{37}$ J. Garcia, C. Challis, J. Citrin, H. Doerk, G. Giruzzi, T. Görler, F. Jenko, P. Maget, and JET Contributors, "Key impact of finite-beta and fast ions in core and edge tokamak regions for the transition to advanced scenarios," Nucl. Fusion 55(5), 053007 (2015).

${ }^{38}$ C. Estrada-Mila, J. Candy, and R. E. Waltz, "Turbulent transport of alpha particles in reactor plasmas," Phys. Plasmas 13(11), 112303 (2006).

${ }^{39}$ J. Citrin, F. Jenko, P. Mantica, D. Told, C. Bourdelle, R. Dumont, J. Garcia, J. W. Haverkort, G. M. D. Hogeweij, T. Johnson et al., "Ion temperature profile stiffness: Non-linear gyrokinetic simulations and comparison with experiment," Nucl. Fusion 54(2), 023008 (2014).

${ }^{40}$ Z. Lin, S. Ethier, T. S. Hahm, and W. M. Tang, "Size scaling of turbulent transport in magnetically confined plasmas," Phys. Rev. Lett. 88, 195004-195Apr (2002).
${ }^{41}$ J. Candy, R. E. Waltz, and W. Dorland, "The local limit of global gyrokinetic simulations," Phys. Plasmas 11(5), L25-L28 (2004).

${ }^{42}$ T. Görler, X. Lapillonne, S. Brunner, T. Dannert, F. Jenko, S. K. Aghdam, P. Marcus, B. F. McMillan, F. Merz, O. Sauter, D. Told, and L. Villard, "Flux- and gradient-driven global gyrokinetic simulation of tokamak turbulence," Phys. Plasmas 18(5), 056103 (2011).

${ }^{43}$ L. Villard, A. Bottino, S. Brunner, A. Casati, J. Chowdhury, T. Dannert, R. Ganesh, X. Garbet, T. Görler, V. Grandgirard, R. Hatzky, Y. Idomura, F. Jenko, S. Jolliet, S. Khosh Aghdam, X. Lapillonne, G. Latu, B. F. McMillan, F. Merz, Y. Sarazin, T. M. Tran, and T. Vernay, "Gyrokinetic simulations of turbulent transport: Size scaling and chaotic behaviour," Plasma Phys. Controlled Fusion 52(12), 124038 (2010).

${ }^{44}$ J. W. Connor, J. B. Taylor, and H. R. Wilson, "Shear damping of drift waves in toroidal plasmas," Phys. Rev. Lett. 70(12), 1803-1805 (1993).

${ }^{45}$ J. Y. Kim and M. Wakatani, "Radial structure of high-mode-number toroidal modes in general equilibrium profiles," Phys. Rev. Lett. 73(16), 2200-2203 (1994).

${ }^{46} \mathrm{~F}$. Romanelli and F. Zonca, "The radial structure of the ion-temperaturegradient-driven mode,” Phys. Fluids B: Plasma Phys. 5(11), 4081-4089 (1993). 